\title{
Plankton-Based Assessment of the Trophic State of Three Tropical Lakes
}

\author{
Benedict Obeten Offem, Ezekiel Olatunji Ayotunde, Gabriel Ujong Ikpi, F. B. Ada, \\ Steven Ncha Ochang
}

Department of Fisheries and Aquatic Sciences, Cross River University of Technology Obubra Campus, Obubra, Nigeria. Email: benbeff06@yahoo.com

Received January $5^{\text {th }}, 2011$; revised February $19^{\text {th }}, 2011$; accepted March $31^{\text {st }}, 2011$.

\begin{abstract}
In developing countries, lakes being important sources of water supply and fishing are vulnerable to anthropogenic impact, yet knowledge of their trophic state in relation to changes in species composition, and environmental variables, are limited. This study is aimed at assessing the trophic status of lakes by monthly sampling of three lakes located along the floodplain of Cross River, Nigeria between January 2008 and December 2009. Samples were analyzed for water quality parameters, zooplankton and phytoplankton composition and distribution. Results were subjected to community structure analysis using trophic state index, species richness and diversity indexes. Essential primary productivity nutrients, nitrates, sulphates and phosphates were highest in Ejagham Lake, and lowest in Ikot Okpora Lake. Dominant phytoplankton species Oscillatoria lacustria (Cyanophyceae), Cyclotella operculata (Bacilliarophyceae) and zooplankton Keratella tropica, Keratella quadrata, Filinia longiseta, Branchionus anguillaris and Trichocerca pusilla (rotifers) all typical of eutrophic communities were recorded in high densities in Ejagham Lake in both dry and wet seasons while Cladocerans, Bosmina longirostris and Moina micrura and copepods considered indicators of oligotrophy and mesotrophy were recorded in large numbers in Ikot Okpora and Obubra Lakes respectively. Higher values of species richness, Evenness and Shannon-Wiener diversity index for both phytoplankton and zooplankton, were recorded in Ejagham Lake during the dry season than wet. Also values of the Trophic state index were generally highest at the Ejagham Lake in the savanna region of the floodplain and lowest at Ikot Okpora in the forest region of the floodplain. Forest region is therefore a limiting factor in the productivity of lakes in the tropics.
\end{abstract}

Keywords: Zooplankton, Phytoplankton, Trophic State Index, Diversity Indices, Species Richness

\section{Introduction}

Biological approaches to evaluating water quality involve assessing communities of organisms. "The basis for this approach is that different species have varying tolerances to environmental stressors" [1]. Fish productivity of water bodies is connected to primary production by many intermediate trophic links. The four groups of organisms that appear in The European Water Framework Directives WFD (Phytoplankton, Zooplankton, Fish and macrophytes), represent water ecological structure over a range of temporal and spatial scales and functional roles. It was recommended that the above biological indicators and, in addition to a range of supporting hydro-morphological and physico-chemical elements should form the core of any monitoring program on lakes [2]. Seasonal changes in mean temperature, radiations, hydrology and nutrient availability are the most impor- tant variables which determine plankton abundance [3]. Also the qualitative and quantitative estimates of the plankton provide good indices of quality and productive capacity of water.

The estimation of phytoplankton density, productivity and trophic status of lakes is very important for fisheries management especially in Nigeria because of dominant tilapia fish culture. The trophic status of a water body is usually estimated by values of primary production measured for the growing season. Classification of lakes based on quantitative trophic indicators such as phosphorus $(\mathrm{P})$ concentration, chlorophyll-a and transparency also allow the trophic status of lakes on a large gradient to be defined [4]. However, these parameters are not always relevant for short-term evolution within any given lake, especially when variations in phosphorus concentration are low. The values of chlorophyll- $a\left(\mu \mathrm{g} \cdot 1^{-1}\right)$ are mostly used as the basic criteria, because it is relatively 
easy and inexpensive to measure and it is a generally used biological measure of phytoplankton biomass and relatively few measurements are needed to get reliable mean value [5]. Carlson's Trophic State Index (TSI) classification [6] can be used to provide a single trophic criterion for the purpose of classifying and ranking water bodies in complex multi-wetland systems. Secchi depth is a much debated and used variable in lake management. Secchi disk transparency is a standard indicator of water clarity, which is strongly correlated with biomass and annual productivity of suspended algae. This is also closely related to the amount of sandy clay, detritus and organic and inorganic suspended and dissolved matter in water [7].

The use of zooplankton community structure as indicator of the wellbeing of water body dates back to 18791910 [8]. Zooplankton is an important component of the trophic food webs of lakes because of its particular position at the crossroads of carbon and energy flows from the lower levels of food chains to fish. Zooplankton biomass which is part of secondary production of lakes is bottom-up regulated by the availability of bacteria and phytoplankton as food and top-down controlled by predation from fish etc. [9]. The composition of zooplankton especially in relation to filter feeders depends on the quality of nutrient supply. So some zooplankton species (mainly rotifers, branchiopods and copepods) could be used as indicators of lakes trophic status [10] because their composition is affected by any of the several environmental parameters e.g. $\mathrm{pH}$ or alkalinity and salinity and other biological parameters [11-13]. Zooplankton abundance is usually closely related to phytoplankton concentration and species composition and increases with increasing nutrients concentrations [14].

Biodiversity is also one of the promising ecological criteria that could be added to lake monitoring programmes. Plankton richness within lakes appears to be largely controlled by factors related to productivity, water quality and fish predation levels. Diversity indices, such as Shannon-Weaner index appeared to detect significant differences in the structure of the communities.

Around the world several researches have been carried out using phytoplankton and zooplankton to investigate pollution [15-19] because they are relatively easy to identify, particularly when community sensitivity can be detected based on plankton body sizes or gross taxonomic classifications. Eastern Nigerian lakes in particular being important sources of water for drinking, fishing and domestic use are vulnerable to anthropogenic impact, yet there is limited water quality data $[20,21]$. This is the first-ever baseline study of the condition of the nation's lakes in eastern Nigeria using statistically valid approach.
It will help the government of the region implement lake monitoring and assessment programs, establish a baseline for lake condition that can be used for future trend assessment. It is focused on studying plankton diversity and evaluating trophic status of some wetlands of Eastern Nigeria for the first time.

\section{Materials and Methods}

\subsection{Study Site}

The Cross River, a floodplain river in south-eastern Nigeria between latitude $4^{\circ} 25^{\prime}-7^{\circ} 00^{\prime} \mathrm{N}$ and longitude $7^{\circ} 15^{\prime}$ $9^{\circ} 30^{\prime} \mathrm{E}$, is bounded in the south by the Atlantic Ocean, east by the Republic of Cameroun and the Nigerian states of Benue in the north, Ebonyi and Abia in the west and Akwa Ibom in the south-west (Figure 1). The climate of the study area comprises a wet season (April- October) characterized by high precipitation $(3050 \pm 230 \mathrm{~mm})$ and a dry season (November - March) marked by low precipitation $(300 \pm 23 \mathrm{~mm})$ [22]. Temperatures range from $15.5^{\circ} \mathrm{C} \pm 7.6^{\circ} \mathrm{C}$ in the wet season to $32.6^{\circ} \mathrm{C} \pm 5.4^{\circ} \mathrm{C}$ in the dry season [20]. Along the floodplain of Cross River are located series of wetlands consisting of lakes, ponds and swamps. The main source of water for these lakes is rainfall and flood water from the river. For the purpose of this study, three lakes were randomly selected along the floodplain of The Cross River, one each from upper section of the floodplain (Ejagham lake), middle portion (Obubra Lake) and down section (Ikot Okpora Lake). Ikot Okpora Lake covers an area of 4 hectares and has maximum depth of $8 \mathrm{~m}$ with a muddy substratum. The lake is used mainly for fishing and has a shoreline thickly shaded by rainforest preventing the effect of direct sunlight rays on the lake water. The lake was almost covered by water hyacinth at the peak of wet season. Bamboo (Bambusa bambusa), Palm trees (E. guinensis) and some other trees characteristics of typical rainforest were present. There was palm oil processing mill near the station and common human activities included drinking, bathing, washing and fishing. Obubra Lake had a rocky, gravel and sandy substratum and covers 4 hectares with maximum depth of $6 \mathrm{~m}$ with shoreline sparsely shaded by forest and savanna grassland. Ejagham Lake, has a sandy and silt dominated substratum opened to direct effect of sunlight ray and shoreline dominated by dense macro-flora Ipomoea pestigridis, Monechma ciliatum, Eragrostic pilosa and Setaria pallide-fusia. The most visible human activities and excavation of sand.

\subsection{Sampling Techniques}

Monthly sampling of the three lakes was carried out from January 2008 to December 2009 at the middle of every 


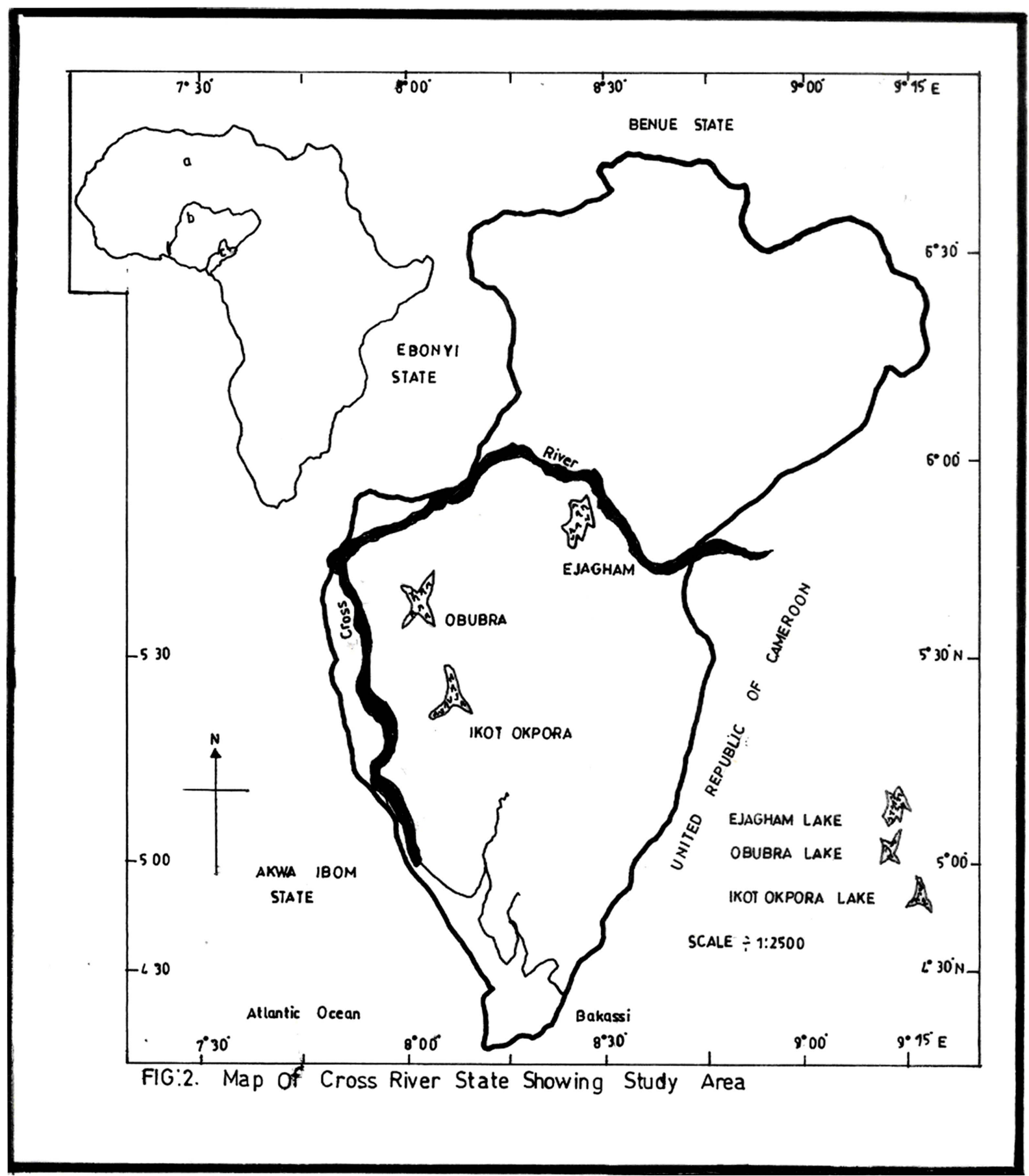

Figure 1. Map of Cross River State showing sampling sites (Source: Cross River State Ministry of lands and survey).

month, between 8 a.m. and 11 a.m. every sampling day.

\subsection{Water Quality Parameters}

Temperature values were recorded from a mercury-in glass thermometer graduated in units of ${ }^{\circ} \mathrm{C}$ by immersing the thermometer slightly under the surface of water $(2$ $\mathrm{cm})$ for 5 minutes until mercury stood at one place). Pye Unicam Model 7065 electronic metre at $25^{\circ} \mathrm{C}$ after stan- dardization with buffer solution at $\mathrm{pH} 4,7$ and 9 was used for $\mathrm{pH}$. Dissolved oxygen concentration of the water samples was determined with a Jen-way 9071 digital oxygen analyzer. Water transparency was measured by use of Secchi disc [23]. The disc was lowered into water and the depth at which it became invisible was recorded. It was then gradually withdrawn from the water and the depth at which it became visible was noted. The trans- 
parency of the water at that station was the mean of the two readings. For the total dissolved solids (TDS), the Hach TDS meter was put on, the reading zeroed and then the electrode dipped into the water sample and the reading taken. Conductivity was assessed by putting on the Suntex conductivity meter, adjusting the reading portion and dipping the meter into the water sample and approximate reading taken. Total hardness, free Carbon dioxide, Acidity, Chemical Oxygen Demand, was obtained by titrimetric method [24]. Biological Oxygen Demand was determined by difference between initial and final dissolved oxygen after incubation for 5 days at room temperature of $\left(20^{\circ} \mathrm{C}\right)$. Total alkalinity was measured by titrating water samples with sulphuric acid standard solution, using a drop of phenolphthalein solution and one sachet of bromcresol green-methyl red as indicator, until the sample changed from blue green to pink. Total alkalinity which is expressed in $\mathrm{mg} / \mathrm{L}$ is the total number of drops of sulphuric acid solution used multiplied by 17.1 (Fish Farmers' Water Quality Testing Kit Manual, 1990). Nitrate and Phosphate were measured with brucine and Ascorbic acid methods respectively. Salinity was determined using hand held refractometer (S/mill-E 0-100\%). Bicarbonate ion was measured with $\mathrm{pH}$ electrode dipped into the tip of the conical container. Sample water was passed through until a constant reading was obtained and a marble powder was added, to completely cover the electrode ball. After about 2 minutes, the $\mathrm{pH}$ was read again. The temperature of the marble was monitored with thermometer during measurement. Carbonate ions was determine by placing hydrochloric acid, between 2 and $10 \mathrm{ml}$ of water sample in a gas generator and insert tube filled with $20 \mathrm{ml}$ hydrochloric acid (10\%). After connecting to the apparatus, the graduated tube was filled by raising the level container. The gas generator was then tilted so that the hydrochloric acid makes contact with the floor. A pressure compensation was attained by sinking the container so that, after about 10 minutes the gas volume can be read. Color was determine by assembling Filter apparatus (membrane filter, filter holder, aspirator and folter flask) and allowed about $50 \mathrm{ml}$ of dematerialized water to pass through to rinse the unit. Discard rinse water. Approximately $50 \mathrm{ml}$ of water sample was filtered and $25 \mathrm{ml}$ poured into another clean cell. The dematerialized water was placed in a cell holder and sample compartment door was closed. The demineralization was used to set the zero concentration point.

\subsection{Phytoplankton}

Planktons were collected in sterilized, one-litre wide mouth dark colour plastic bottles at each sampling station, reduced to $10 \mathrm{ml}$ by decanting the supernatant aliquot and preserved with Lugol's solution. Samples were shifted to laboratory where they were identified after Prescott [25], Edmondson [26] and John et al. [27]. For Chlorophyll-a analysis $100 \mathrm{ml}$ water sample were filtered through Millipore micro filters (47 mm; $45 \mu \mathrm{m}$ pores). Concentration of Chlorophyll-a in supernatant was determined by spectrophotometer, with absorbance at 665 $\mathrm{nm}$ and $750 \mathrm{~nm}$ [28].

\subsection{Zooplankton}

Zooplankton was collected by towing a-55 $\mu$ mesh-sized plankton net against the current flow at the subsurface level for two minutes [29]. The filtered samples were washed into the sterilized collecting bottles and immediately fixed in $4 \%$ formalin. The percentage relative abundance of the specimen was estimated by irect count. Each quantitative sample was concentrated to $10 \mathrm{ml}$ and from this; $1 \mathrm{ml}$ of sample was taken and all individual taxa present were counted. Relative abundance was calculated as the number of individuals per liter of water filtered though the net. They were identified with an Olympus Vanox Research Microscope (mag X60) Model 230485 using keys given by Kadiri [30] and Kemdirim [31].

\subsection{Community Structure Analysis}

Trophic state index based on Secchi Depth TSI (SD) and Trophic state index based on Chlorophyll-a, TSI (Chl-a) and that based on phosphorus, TSI(TP) were calculated after Carlson [32] using the following equations:

TSI $($ Secchi Disk $)=60-14.1$ In (SD) where SD is Secchi Disk in meters(m)

TSI $($ Chlorophyll a $)=9.81($ In Chl-a $)+30.6$ where Chl a is mean chlorophyll a in $\mu \mathrm{g} \cdot \mathrm{l}^{-1}$ TSI (Total Phosphorus) $=$ $14.41 \ln (\mathrm{TP})+4.15$ where TP is mean total phosphorus in $\mu \mathrm{g} \cdot 1^{-1}$.

Diversity indices used were species diversity $\left(\mathrm{H}^{\prime}\right)$, species richness (d) and species evenness. Shannon-Weaner diversity function was used to calculate heterogeneity for each site. This index takes into account the total number of species present as well as their respective abundance thus providing a more convenient means of comparing differences between ecological communities. These changes in the environment are reflected in the types and number of organisms.

Richness index was expressed using Margelef's richness index. This measure relies only on the number of taxa. Richness increases when abundance is spread over a greater number of categories but does not take into account the evenness of the distribution. Also between two samples with the same $\mathrm{S}$, richness will be higher in 
the one with lower abundance. Evenness Index, which expresses the degree of uniformity in the distribution of individual among the taxa in the collection [33] was also calculated.

$$
\begin{aligned}
& d=(\mathrm{S}-1) / \operatorname{logN}(\text { Equation } 1) \\
& H=-\mathrm{Sp}_{i}\left(\log \mathrm{p}_{i}\right)(\text { Equation }) \\
& E=\mathrm{H} / \mathrm{SE}=\text { Equitability } \\
& d=\text { Margalef's richness index and } \mathrm{H}^{\prime}=\text { Shanon-Wiener } \\
& \text { Diversity Function } \\
& S=\text { total species number } \\
& \mathrm{p}_{i}=\text { proportion of each species in each sample, }
\end{aligned}
$$

besides the application of diversity indices, inter-stations comparison were carried out to test for significant differences in faunal abundance using one-way analysis of variance (ANOVA) [34].

\section{Result}

\subsection{Environmental Conditions}

As shown in Table 1, significant spatial variation with higher values $(p<0.05)$ was observed for air temperature, surface temperature, turbidity, alkalinity, conductivity, BOD, phosphates, sulphates, chlorides, calcium, magnesium, silicon, total hardness, total solids and total dissolved solids in the Ejagham Lake than Obubra and Ikot Okpora Lakes while Dissolved Oxygen and alkalinity were least in Ejagham Lake. Other variables showed no significant spatial differences $(\mathrm{p}>0.05)$.

\subsection{Phytoplankton}

A total of 46 planktonic algae from 5 taxa were recorded: 17 Bacillariophyceae, 19 Chlorophyceae, 8 Cyanophyceae, 1 Chrysophyceae and 1 Euglanaphyceae (Table 2). There was significant seasonal and spatial variation in the phytoplankton abundance and distribution. Apart from Rhyzosolenia longiseta, Melosera varians, Cyclotella operculata, Chlorococcum humicolum, Closteridum lanceolatum Golonotozygon aculeatum, Sphaerocystis species, Raphidiopsis species, Epithermal zebra, Chlamydomonas atactogam, Closteriopsis longissinna, Golonotozygon aculeatum Schizoponium praisiola, Tetrahedron species, Spirulina substilissinna, Colactum cyclopicola, Closterium junoidum and Golonotozygon aculeatum that had higher wet season counts, all other species in the three lakes had higher dry season counts. The highest number of algal counts was recorded in the Ejagham Lake while the least occurred in Ikot Okporo. Cocconeis dimunuta, Oscilatoria Lacustria and Dinobryon species were however completely absent

\begin{tabular}{|c|c|c|c|}
\hline Parameters & Ikot Okpora & Obubra & Ejagham \\
\hline Total Storage $\left(\mathrm{m}^{3}\right)$ & $638^{\mathrm{a}}$ & $1099^{\mathrm{b}}$ & $1236^{\mathrm{b}}$ \\
\hline Lake Area (ha) & $45.4 \pm 8.76^{\mathrm{a}}$ & $51.3 \pm 5.66^{\mathrm{a}}$ & $76.4 \pm 12.34^{\mathrm{b}}$ \\
\hline Mean Depth (m) & $8.2 \pm 2.81^{\mathrm{a}}$ & $7.6 \pm 3.23^{\mathrm{a}}$ & $8.9 \pm 2.45^{\mathrm{a}}$ \\
\hline Air Temperature $\left({ }^{\circ} \mathrm{C}\right)$ & $30.9 \pm 2.23^{\mathrm{a}}$ & $32.5 \pm 1.84^{\mathrm{a}}$ & $34.4 \pm 3.42^{\mathrm{b}}$ \\
\hline Surface Temperature $\left({ }^{\circ} \mathrm{C}\right)$ & $27.7 \pm 1.71^{\mathrm{a}}$ & $29.4 \pm 2.11^{\mathrm{a}}$ & $33.8 \pm 2.89^{b}$ \\
\hline Turbidity(NTU) & $12.5 \pm 2.23^{\mathrm{a}}$ & $66.9 \pm 6.33^{\mathrm{b}}$ & $132.4 \pm 23.34^{\mathrm{c}}$ \\
\hline $\mathrm{Ph}$ & $6.5 \pm 0.66^{\mathrm{a}}$ & $7.2 \pm 1.12^{\mathrm{a}}$ & $6.8 .5 \pm 1.81^{\mathrm{a}}$ \\
\hline Dissolved Oxygen $\left(\mathrm{mg} \cdot 1^{-1}\right)$ & $3.2 \pm 1.21^{\mathrm{a}}$ & $2.7 \pm 0.89^{b}$ & $2.3 \pm 0.76^{\mathrm{c}}$ \\
\hline Alkalinity $\left(\mathrm{mg} \cdot \mathrm{1}^{-1}\right)$ & $53.2 \pm 4.42^{\mathrm{a}}$ & $40.8 \pm 5.43^{\mathrm{b}}$ & $67.4 \pm 12.98^{\mathrm{c}}$ \\
\hline Conductivity $\left(\mu \mathrm{S} \cdot \mathrm{cm}^{-1}\right)$ & $22.4 \pm 14.78^{\mathrm{a}}$ & $87.6 \pm 10.32^{b}$ & $102.8 \pm 16.78^{\mathrm{c}}$ \\
\hline $\mathrm{BOD}\left(\mathrm{mg} \cdot \mathrm{l}^{-1}\right)$ & $1.4 \pm 1.66^{\mathrm{a}}$ & $2.6 \pm 0.87^{b}$ & $4.5 \pm 1.64^{\mathrm{c}}$ \\
\hline Phosphates (mg/l) & $0.5 \pm 0.01^{\mathrm{a}}$ & $1.5 \pm 0.04^{\mathrm{b}}$ & $2.5 \pm 0.23^{\mathrm{c}}$ \\
\hline Calcium $\left(\mathrm{Ca}^{2+}\right)$ & $10.4 \pm 3.4^{\mathrm{a}}$ & $9.3 \pm 2.87^{\mathrm{a}}$ & $19.4 \pm 4.83^{\mathrm{b}}$ \\
\hline Magnesium $\left(\mathrm{Mg}^{2+}\right)$ & $1.8 \pm 0.22^{\mathrm{a}}$ & $2.1 \pm 0.54^{\mathrm{a}}$ & $3.2 \pm 0.99^{\mathrm{b}}$ \\
\hline Total iron & $1.4 \pm 0.56^{\mathrm{a}}$ & $0.7 \pm 0.43^{\mathrm{a}}$ & $1.9 \pm 0.36^{\mathrm{a}}$ \\
\hline Silicon $\left(\mathrm{SiO}_{2}\right)$ & $38.9 \pm 2.89^{\mathrm{a}}$ & $28.4 \pm 5.34^{\mathrm{b}}$ & $56.8 \pm 9.65^{\mathrm{c}}$ \\
\hline Sulphates $\left(\mathrm{SO}_{4}\right)$ & $11.4 \pm 1.23^{\mathrm{a}}$ & $14.8 \pm 2.88^{\mathrm{a}}$ & $19.8 \pm 7.81^{\mathrm{b}}$ \\
\hline Nitrates $\left(\mathrm{NO}_{3}\right)$ & $0.2 \pm 0.78^{\mathrm{a}}$ & $0.4 \pm 0.34^{b}$ & $0.8 \pm 0.56^{\mathrm{a}}$ \\
\hline Chlorides $(\mathrm{Cl})$ & $13.6 \pm 5.99^{\mathrm{a}}$ & $11.6 \pm 3.21^{\mathrm{a}}$ & $18.8 \pm 6.77^{\mathrm{b}}$ \\
\hline Total hardness & $57.5 \pm 8.88^{\mathrm{a}}$ & $94.3 \pm 6.54^{\mathrm{b}}$ & $143.5 \pm 23.56^{\mathrm{c}}$ \\
\hline Total solids (mg/l) & $68.9 \pm 12.34^{\mathrm{a}}$ & $187.6 \pm 8.76^{\mathrm{b}}$ & $208.3 \pm 54.50^{\mathrm{c}}$ \\
\hline Total Dissolved solids (mg/l) & $59.8 \pm 6.54^{\mathrm{a}}$ & $178.5 \pm 6.66^{\mathrm{b}}$ & $222.8 \pm 65.44^{\mathrm{c}}$ \\
\hline
\end{tabular}
from the Ejagham Lake. The observed dominant species in the Ejagham Lake, during both wet and dry seasons was Oscillatoria lacustria (Cyanophyceae) closely followed by Cyclotella operculata (Bacilliarophyceae) while

Table 1. Mean ( \pm SD) values of some physical and chemical characteristics of the three lakes (Ikot Okpora, Obubra and Ejagham).

Values in the same rows with the same superscript are not significantly different $(p>0.05)$. 
Table 2. Phytoplankton of Ikot Okpora, Obubra and Ejagham Lakes during wet (W) and dry (D) seasons.

\begin{tabular}{|c|c|c|c|c|c|c|}
\hline Season & .Okpora W & $\mathrm{D}$ & Obubra W & $\mathrm{D}$ & Ejagham W & $\mathrm{D}$ \\
\hline Phytoplankton Species & $\mathrm{No} / \mathrm{ml}$ & $\mathrm{No} / \mathrm{ml}$ & $\mathrm{No} / \mathrm{ml}$ & $\mathrm{No} / \mathrm{ml}$ & $\mathrm{No} / \mathrm{ml}$ & $\mathrm{No} / \mathrm{ml}$ \\
\hline \multicolumn{7}{|l|}{ Bacillariophyceae } \\
\hline Tabellaria Flocculosa & 34 & 49 & 87 & 192 & 134 & 234 \\
\hline Synedra Cyclopum & 00 & 65 & 09 & 87 & 26 & 198 \\
\hline Suriellia Spiralis & 00 & 50 & 00 & 76 & 00 & 86 \\
\hline Rhyzosolenia Longiseta & 122 & 36 & 156 & 48 & 222 & 433 \\
\hline Melosera Varians & 134 & 88 & 223 & 176 & 320 & 482 \\
\hline Navicula Rostellata & 00 & 98 & 102 & 267 & 243 & 543 \\
\hline Nitzschia Closterium & 89 & 101 & 112 & 344 & 176 & 410 \\
\hline Cytosigma Attenuatum & 67 & 45 & 136 & 233 & 267 & 309 \\
\hline Flagilaria Intermedia & 94 & 222 & 87 & 342 & 120 & 654 \\
\hline Epithermal Zebra & 34 & 98 & 243 & 132 & 298 & 499 \\
\hline Cocconeis Dimunuta & 00 & 76 & 00 & 67 & 00 & 00 \\
\hline Cymatopleura Solea & 65 & 98 & 98 & 200 & 110 & 222 \\
\hline Cyclotella Glomerata & 76 & 118 & 99 & 199 & 230 & 349 \\
\hline Cyclotella Comta & 00 & 223 & 87 & 322 & 167 & 276 \\
\hline Cyclotella Operculata & 816 & 1464 & 511 & 1389 & 624 & 1195 \\
\hline Amphora Ovalis & 222 & 553 & 87 & 627 & 187 & 299 \\
\hline Amphilpleura Pellicuda & 98 & 112 & 202 & 322 & 324 & 562 \\
\hline \multicolumn{7}{|l|}{ Chlorophyceae } \\
\hline Spirogyra sp & 223 & 655 & 127 & 498 & 242 & 576 \\
\hline Chlorococcum Humicolum & 232 & 98 & 299 & 456 & 342 & 399 \\
\hline Chlamydomonas Atactogam & 67 & 633 & 276 & 202 & 433 & 00 \\
\hline Closteriopsis Longissinna & 87 & 87 & 123 & 00 & 234 & 488 \\
\hline Codiolum Gregarii & 99 & 211 & 97 & 110 & 178 & 876 \\
\hline Closterium Jennen & 77 & 199 & 88 & 290 & 134 & 489 \\
\hline Drapamidia Species & 00 & 120 & 155 & 388 & 209 & 119 \\
\hline Neomens Dumetosa & 72 & 432 & 87 & 282 & 198 & 198 \\
\hline Charales Fragilis & 00 & 87 & 54 & 389 & 123 & 467 \\
\hline Shroeederia Setigera & 39 & 108 & 98 & 98 & 176 & 198 \\
\hline Closterium Junoidum & 00 & 56 & 63 & 187 & 233 & 67 \\
\hline Closteridum Lanceolatum & 95 & 73 & 59 & 78 & 00 & 78 \\
\hline Golonotozygon Aculeatum & 66 & 18 & 167 & 56 & 234 & 378 \\
\hline Schizoponium Praisiola & 101 & 193 & 91 & 21 & 145 & 576 \\
\hline Serastrium Spinolosum & 56 & 198 & 66 & 134 & 133 & 366 \\
\hline Spirotaenna Condensate & 78 & 97 & 102 & 167 & 187 & 266 \\
\hline Volvox Aurus & 39 & 67 & 23 & 101 & 43 & 102 \\
\hline Sphaerocystis Species & 100 & 44 & 00 & 122 & 00 & 736 \\
\hline Tetrahedron Species & 00 & 194 & 233 & 65 & 129 & 234 \\
\hline \multicolumn{7}{|l|}{ Cyanophyceae } \\
\hline Oscilatoria Lacustria & 12 & 23 & 54 & 32 & 1878 & 2842 \\
\hline Pharmidium Species & 101 & 134 & 55 & 71 & 134 & 1120 \\
\hline Spirulina Substilissinna & 00 & 134 & 129 & 76 & 489 & 1564 \\
\hline Rivularia Plankton & 81 & 234 & 31 & 92 & 87 & 342 \\
\hline Anabaena Species & 0 & 67 & 78 & 102 & 110 & 1290 \\
\hline Microcystis Species & 230 & 1222 & 188 & 981 & 1558 & 2733 \\
\hline Anabaenopsis Species & 0 & 26 & 83 & 233 & 134 & 556 \\
\hline Raphidiopsis Species & 33 & 0 & 45 & 00 & 987 & 1234 \\
\hline \multicolumn{7}{|l|}{ Chrysophyceae } \\
\hline Dinobryon Species & 1 & 2 & 0 & 12 & 0 & 0 \\
\hline \multicolumn{7}{|l|}{ Euglenaphyceae } \\
\hline Colactum Cyclopicola & 00 & 32 & 34 & 0 & 78 & 324 \\
\hline
\end{tabular}


Table 3. Seasonal variation of phytoplankton diversity indices in Ikot Okpora, Obubra and Ejagham Lakes.

\begin{tabular}{|c|c|c|c|c|c|c|}
\hline Lakes & Phytoplankton & Seasons & Dorminance & Evenness & Margalef & Shan non \\
\hline \multirow{10}{*}{ Ikot Okpora } & \multirow{2}{*}{ Bacillariophyceae } & Wet & $0.17 \pm 0.03^{\mathrm{a}}$ & $0.76 \pm 0.13^{\mathrm{a}}$ & $3.24 \pm 0.12^{\mathrm{a}}$ & $0.45 \pm 0.17^{\mathrm{a}}$ \\
\hline & & Dry & $0.43 \pm 0.02^{b}$ & $0.56 \pm 0.34^{\mathrm{b}}$ & $3.97 \pm 0.23^{\mathrm{a}}$ & $0.95 \pm 0.22^{b}$ \\
\hline & \multirow{2}{*}{ Chlorophyceae } & Wet & $0.22 \pm 0.01^{\mathrm{a}}$ & $0.66 \pm 0.04^{\mathrm{a}}$ & $3.45 \pm 0.21^{\mathrm{a}}$ & $0.59 \pm 0.14^{\mathrm{a}}$ \\
\hline & & Dry & $0.48 \pm 0.02^{\mathrm{b}}$ & $0.58 \pm 0.11^{\mathrm{b}}$ & $3.89 \pm 0.33^{\mathrm{a}}$ & $0.89 \pm 0.28^{b}$ \\
\hline & \multirow{2}{*}{ Cyanophyceae } & Wet & $0.13 \pm 0.15^{\mathrm{a}}$ & $0.81 \pm 0.13^{\mathrm{a}}$ & $2.31 \pm 0.19^{\mathrm{a}}$ & $0.36 \pm 0.19^{\mathrm{a}}$ \\
\hline & & Dry & $0.35 \pm 0.11^{b}$ & $0.61 \pm 0.23^{\mathrm{b}}$ & $2.81 \pm 0.26^{\mathrm{a}}$ & $0.66 \pm 0.28^{a}$ \\
\hline & \multirow{2}{*}{ Chrysophyceae } & Wet & $0.00 \pm 0.00$ & $0.00 \pm 0.00$ & $0.00 \pm 0.00$ & $0.00 \pm 0.00$ \\
\hline & & Dry & $0.00 \pm 0.00$ & $0.00 \pm 0.00$ & $0.00 \pm 0.00$ & $0.00 \pm 0.00$ \\
\hline & \multirow{2}{*}{ Euglenaphyceae } & Wet & $0.00 \pm 0.00$ & $0.00 \pm 0.00$ & $0.00 \pm 0.00$ & $0.00 \pm 0.00$ \\
\hline & & Dry & $0.00 \pm 0.00$ & $0.00 \pm 0.00$ & $0.00 \pm 0.00$ & $0.00 \pm 0.00$ \\
\hline \multirow{9}{*}{ Obubra } & \multirow{2}{*}{ Bacillariophyceae } & Wet & $0.12 \pm 0.01^{\mathrm{a}}$ & $0.91 \pm 0.10^{\mathrm{a}}$ & $3.76 \pm 0.17^{\mathrm{a}}$ & $1.03 \pm 0.15^{\mathrm{a}}$ \\
\hline & & Dry & $0.18 \pm 0.03^{b}$ & $0.89 \pm 0.19^{b}$ & $3.97 \pm 0.29^{\mathrm{a}}$ & $1.76 \pm 0.21^{b}$ \\
\hline & \multirow{2}{*}{ Chlorophyceae } & Wet & $0.29 \pm 0.05^{\mathrm{a}}$ & $0.66 \pm 0.21^{\mathrm{a}}$ & $3.91 \pm 0.19^{\mathrm{a}}$ & $1.05 \pm 0.33^{\mathrm{a}}$ \\
\hline & & Dry & $0.17 \pm 0.11^{b}$ & $0.79 \pm 0.11^{\mathrm{b}}$ & $4.11 \pm 0.26^{\mathrm{a}}$ & $1.88 \pm 0.29^{b}$ \\
\hline & \multirow{2}{*}{ Cyanophyceae } & Wet & $0.23 \pm 0.06^{\mathrm{a}}$ & $0.82 \pm 0.17^{\mathrm{a}}$ & $3.11 \pm 0.22^{\mathrm{a}}$ & $0.68 \pm 0.16^{\mathrm{a}}$ \\
\hline & & Dry & $0.44 \pm 0.13^{\mathrm{b}}$ & $0.54 \pm 0.29^{\mathrm{b}}$ & $3.88 \pm 0.16^{\mathrm{a}}$ & $1.22 \pm 0.19^{b}$ \\
\hline & Chrysophyceae & Dry & $0.00 \pm 0.00$ & $0.00 \pm 0.00$ & $0.00 \pm 0.00$ & $0.00 \pm 0.00$ \\
\hline & \multirow{2}{*}{ Euglenaphyceae } & Wet & $0.00 \pm 0.00$ & $0.00 \pm 0.00$ & $0.00 \pm 0.00$ & $0.00 \pm 0.00$ \\
\hline & & Dry & $0.00 \pm 0.00$ & $0.00 \pm 0.00$ & $0.00 \pm 0.00$ & $0.00 \pm 0.00$ \\
\hline \multirow{10}{*}{ Ejagham } & \multirow{2}{*}{ Bacillariophyceae } & Wet & $0.23 \pm 0.16^{\mathrm{a}}$ & $0.84 \pm 0.18^{\mathrm{a}}$ & $3.85 \pm 0.25^{\mathrm{a}}$ & $1.69 \pm 0.33^{\mathrm{a}}$ \\
\hline & & Dry & $0.36 \pm 0.21^{\mathrm{b}}$ & $0.54 \pm 0.32^{\mathrm{b}}$ & $4.32 \pm 0.28^{\mathrm{a}}$ & $2.88 \pm 0.21^{\mathrm{b}}$ \\
\hline & \multirow{2}{*}{ Chlorophyceae } & Wet & $0.18 \pm 0.05^{\mathrm{a}}$ & $0.95 \pm 0.22^{\mathrm{a}}$ & $4.21 \pm 0.14^{\mathrm{a}}$ & $1.55 \pm 0.14^{\mathrm{a}}$ \\
\hline & & Dry & $0.64 \pm 0.29^{b}$ & $0.42 \pm 0.17^{\mathrm{b}}$ & $4.87 \pm 0.21^{\mathrm{a}}$ & $2.97 \pm 0.43^{b}$ \\
\hline & \multirow{2}{*}{ Cyanophyceae } & Wet & $0.540 \pm 0.07^{\mathrm{a}}$ & $0.44 \pm 0.14^{\mathrm{a}}$ & $2.88 \pm 0.24^{\mathrm{a}}$ & $1.69 \pm 0.22^{\mathrm{a}}$ \\
\hline & & Dry & $0.86 \pm 0.16^{\mathrm{b}}$ & $0.29 \pm 0.23^{\mathrm{b}}$ & $3.07 \pm 0.18^{\mathrm{a}}$ & $1.92 \pm 0.19^{\mathrm{a}}$ \\
\hline & \multirow{2}{*}{ Chrysophyceae } & Wet & $0.00 \pm 0.00$ & $0.00 \pm 0.00$ & $0.00 \pm 0.00$ & $0.00 \pm 0.00$ \\
\hline & & Dry & $0.00 \pm 0.00$ & $0.00 \pm 0.00$ & $0.00 \pm 0.00$ & $0.00 \pm 0.00$ \\
\hline & \multirow{2}{*}{ Euglenaphyceae } & Wet & $0.00 \pm 0.00$ & $0.00 \pm 0.00$ & $0.00 \pm 0.00$ & $0.00 \pm 0.00$ \\
\hline & & Dry & $0.00 \pm 0.00$ & $0.00 \pm 0.00$ & $0.00 \pm 0.00$ & $0.00 \pm 0.00$ \\
\hline
\end{tabular}

Means with the same superscript are not significantly different $(p>0.05)$.

the least species recorded was Dinobryon species (Chrysophyceae). There were significant seasonal variations of species diversity indices for all phytoplankton taxa $(\mathrm{p}<0.05)$ except Margalef index none for all the species from the three lakes (Table 3).

\subsection{Zooplankton}

A total of 26 zooplankton species consisting of 4 taxa were recorded (Table 4). Rotifers (9) and Cladocerans (8) had the highest representation by species followed by Copepoda (5) while Decapods were least represented (4). Among the rotifers, Keratella tropica, Keratella quadrata, Filinia longiseta, Branchionus anguillaris and Trichocerca pusilla and cladocerans, Ceriodaphnia cornuta, Chydorus sphaericus and Diaphanosoma excisum were common in Ejagham Lake. Rotifers like Synchaeta longipes and Conochilus dossuarius and Cladocera, Bosmina longirostris and Moina micrura were recorded in high densities in Ikot Okpora Lake. Cyclopoid copepodids and nauphlii increased the population of Copepoda particularly in Ikot Okpora Lake during dry season while the numerical abundance reduced drastically in Ejagham Lake.

Seasonal variation of species diversity indices for zoo- plankton were significant $(\mathrm{p}<0.05)$ for all 3 lakes $($ Table 5). However, seasonal variation of Margalef index was not significant for all species in the 3 lakes. Higher values of species richness, Evenness and Shannon-Wiener diversity index were recorded during the dry season than the wet.

\subsection{Trophic State Index}

In the three lakes studied chlorophyll-a concentration increased in dry season and decrease in the wet. Trophic State Index dry season values based on Chlorophyll-a, Secchi Disk and Phosporus were generally highest at the Ejagham (57.02, 77.22 and 74.40) Lake and lowest at Ikot Okpora (41.21, 49.52 and 60.9) respectively (Table 6). In all the lakes Trophic Index value based on total phosphorus; TSI (TP) is higher $(60.9,63.12,74.4)$ than the values by Chlorophyll-a; TSI (Chl-a) (41.21, 52.43, 56.01) and Secchi disk; TSI (SD) (49.52, 51.11 77.22) respectively.

\section{Discussion}

\subsection{Physico-Chemistry}

Essential primary productivity nutrients, nitrates, sulphates and phosphates were highest in Ejagham Lake, 
Table 4. Zooplankton of Ikot Okpora, Obubra and Ejagham Lakes during wet (W) and dry (D) seasons.

\begin{tabular}{|c|c|c|c|c|c|c|}
\hline Species & $\mathrm{W}$ & $\mathrm{D}$ & $\mathrm{W}$ & $\mathrm{D}$ & $\mathrm{W}$ & $\mathrm{D}$ \\
\hline \multicolumn{7}{|l|}{ Cladocera } \\
\hline Alona rectangula & 59 & 185 & 23 & 40 & 7 & 23 \\
\hline Ceriodaphnia cornuta & 34 & 149 & 87 & 83 & 12 & 13 \\
\hline Chydorus sphaericus & 29 & 254 & 74 & 88 & 3 & 28 \\
\hline Diaphanosoma excisum & 40 & 176 & 2 & 94 & 23 & 13 \\
\hline Simocephalus & 0 & 113 & 15 & 56 & 24 & 89 \\
\hline Moina micrura & 65 & 198 & 24 & 33 & 12 & 44 \\
\hline Bosmina longirostris & 69 & 123 & 30 & 11 & 0 & 0 \\
\hline Macrothrix spinosa & 65 & 12 & 56 & 0 & 122 & 67 \\
\hline Copepoda & & & & & 34 & \\
\hline Ectocyclops phaleratus & 92 & 178 & 34 & 56 & & 41 \\
\hline Mesocyclop notius & 85 & 177 & 33 & 57 & 0 & 48 \\
\hline Merocyclop & 118 & 184 & 19 & 56 & 0 & 10 \\
\hline Metacyclops minutus & 11 & 0 & 8 & 12 & 23 & 45 \\
\hline Microcyclops & 85 & 13 & 23 & 34 & 57 & 78 \\
\hline Decapoda & & & & & 84 & \\
\hline Lucifer hansenii & 45 & 87 & 76 & 124 & & 176 \\
\hline Bipinnaria larva & 18 & 34 & 34 & 76 & 32 & 45 \\
\hline Mysid larvae & 23 & 45 & 34 & 56 & 45 & 121 \\
\hline Diphanosoma excisum & 43 & 87 & 12 & 111 & 98 & 101 \\
\hline Rotifera & 12 & 56 & 23 & 54 & 34 & 333 \\
\hline Keratella tropica & 22 & 44 & 12 & 84 & 23 & 176 \\
\hline Keratella quadrata & 0 & 0 & 7 & 56 & 23 & 66 \\
\hline Epiphanes macronna & 59 & 123 & 43 & 58 & 13 & 83 \\
\hline Trycocerca similis & 62 & 165 & 28 & 42 & 22 & 18 \\
\hline Synchaeta longipes & 112 & 98 & 29 & 66 & 2 & 6 \\
\hline Conochilus dossuarius & 122 & 101 & 34 & 55 & 3 & 8 \\
\hline Trichocerca pusilla & 10 & 12 & 8 & 34 & 23 & 98 \\
\hline Branchionus anguillaris & 23 & 34 & 23 & 56 & 98 & 178 \\
\hline Filinia longiseta & 9 & 45 & 39 & 65 & 67 & 234 \\
\hline
\end{tabular}

surrounded by the savanna vegetation and lowest in Ikot Okpora Lake; the forest portion of the floodplain. Forest ecosystem readily immobilise these nutrients [35], which explains its limiting inputs into the water body in the forest area. The levels of nutrients are fundamental to the eutrophic nature of the lakes. Evidence for high production is also seen in the lakes oxygen profile which went down low $(2.3 \mathrm{mg} / \mathrm{L})$ at the Ejagham Lake, a condition which contributes to the increase of lake phosphorus through the release of phosphorus by deoxidized iron in the sediments. The concentrations of calcium and magnesium salts combined with various anions (usually carbonates) that constitute the total hardness of water were high in Ejagham Lake indicating hard water lake. The lower values of these parameters in Ikot Okpora lake and Obubra showed the soft water nature of these lakes. Lake water was clearer at Ikot Okpora than other lakes due to lower values of turbidity at this forest lake.

\subsection{Phytoplankton}

Although Bacillariophyta and Chlorophyta were dominant in respect to species number, Cyanophyta type phytoplankton were more in terms of population density in Ejagham Lake. Oscilatoria lacustria Microcystis spe- cies, Spirulina substilissinna, Raphidiopsis species were dominant and sub-dominant organisms in certain seasons. Cyanophyta dominance, and sometimes bloom formation are among the most visible symptoms of accelerated euthrophication of lakes and reservoirs [36-38]. Also the permanent dominance of Oscillatoria species during dry and wet seasons has often been reported for eutrophic lakes in Central Europe [39]. The observed dominant species, Cyclotella operculata (Bacillariophyta) by Nwankwo [40] and Microcystis by Silva [18] in both wet and dry season was reported to be as a result of physiological and behavioural flexibility of these species which can accommodate environmental stresses better than most fast growing species.

\subsection{Zooplankton}

Numerous species of rotifers and crustaceans considered good indicators of the trophic state of lakes were found in the zooplankton community. Rotifers recorded frequently in the Ikot Okpora Lake, Synchaeta longipes and Conochilus dossuarius are typical of oligotrophic to mesotrophic systems [41]. However, the regularly most dominant species in the Ejagham Lake like Keratella tropica, Keratella quadrata, Filinia longiseta, Bran 
chionus anguillaris and Trichocerca pusilla are considered indicators of advanced lake trophy [10]. The crustacean zooplankton community was made up of cladocerans and copepods. Copepod abundance in Ikot Okpora and Obubra lakes was driven by the increase of Ectocyclops, Mesocyclops, merocyclops, Microcyclops which are indicative of higher water quality signifying oligotrophic status. Crustacean abundance increases only between mesotrophic and meso-eutrophic [42]. Also dominant cladocerans, Alona rectangular, Ceriodaphnia cornuta, Chydorus sphaericus, Diaphanosoma excisum, Simocephalus sp., Moina micrura and Bosmina longirostris represented in most samples in the Ikot Okpora Lake are well recorded by Sendacz et al. [43] and Swierzowski et al. [44] in oligotrophic and mesooligotrophic systems. Generally increase in the abundance of zooplankton in the Ejagham Lake further confirms the eutrophic status of the lake since zooplankton abundance increases with increasing nutrient concentration [14] and decreases with decreasing nutrient concentration.

Table 5. Seasonal variation of zooplankton diversity indices in Ikot Okpora, Obubra and Ejagham Lakes.

\begin{tabular}{|c|c|c|c|c|c|c|}
\hline Lakes & Zooplankton & Seasons & Dominance & Evenness & Margelef & Shannon \\
\hline \multirow{8}{*}{ Ikot Okpora } & \multirow{2}{*}{ Cladocera } & Wet Season & $0.44 \pm 0.07^{\mathrm{a}}$ & $0.56 \pm 0.12^{\mathrm{a}}$ & $3.56 \pm 0.17^{\mathrm{a}}$ & $0.55 \pm 0.05^{\mathrm{a}}$ \\
\hline & & Dry Season & $0.29 \pm 0.11^{\mathrm{b}}$ & $0.96 \pm 0.16^{\mathrm{b}}$ & $4.87 \pm 0.21^{\mathrm{a}}$ & $0.73 \pm 0.19^{b}$ \\
\hline & \multirow{2}{*}{ Copepoda } & Wet Season & $0.63 \pm 0.09^{\mathrm{a}}$ & $0.51 \pm 0.07^{\mathrm{a}}$ & $2.11 \pm 0.15^{\mathrm{a}}$ & $0.47 \pm 0.18^{\mathrm{a}}$ \\
\hline & & Dry Season & $0.32 \pm 0.22^{\mathrm{b}}$ & $0.87 \pm 0.10^{\mathrm{b}}$ & $2.99 \pm 0.03^{\mathrm{a}}$ & $0.88 \pm 0.14^{b}$ \\
\hline & \multirow{2}{*}{ Decapoda } & Wet Season & $0.58 \pm 0.06^{\mathrm{a}}$ & $0.44 \pm 0.26^{\mathrm{a}}$ & $1.68 \pm 0.22^{\mathrm{a}}$ & $0.23 \pm 0.18^{\mathrm{a}}$ \\
\hline & & Dry Season & $0.20 \pm 0.15^{\mathrm{b}}$ & $0.79 \pm 0.15^{\mathrm{b}}$ & $1.94 \pm 0.18^{\mathrm{a}}$ & $0.44 \pm 0.11^{\mathrm{b}}$ \\
\hline & \multirow{2}{*}{ Rotifera } & Wet Season & $0.76 \pm 0.23^{\mathrm{a}}$ & $0.25 \pm 0.08^{\mathrm{a}}$ & $1.66 \pm 0.11^{\mathrm{a}}$ & $0.36 \pm 0.26^{\mathrm{a}}$ \\
\hline & & Dry Season & $0.51 \pm 0.22^{\mathrm{b}}$ & $0.55 \pm 0.01^{\mathrm{b}}$ & $1.23 \pm 0.20^{\mathrm{a}}$ & $0.69 \pm 0.18^{\mathrm{b}}$ \\
\hline \multirow{7}{*}{ Obubra } & \multirow[t]{2}{*}{ Cladocera } & Wet Season & $0.75 \pm 0.19^{\mathrm{a}}$ & $0.25 \pm 0.11^{\mathrm{a}}$ & $4.81 \pm 0.34^{\mathrm{a}}$ & $0.45 \pm 0.08^{\mathrm{a}}$ \\
\hline & & Dry Season & $0.46 \pm 0.12^{\mathrm{b}}$ & $0.50 \pm 0.02^{\mathrm{b}}$ & $4.33 \pm 0.16^{\mathrm{a}}$ & $0.86 \pm 0.43^{b}$ \\
\hline & \multirow{2}{*}{ Copepoda } & Wet Season & $0.955 \pm 0.18^{\mathrm{a}}$ & $0.11 \pm 0.11^{\mathrm{a}}$ & $3.22 \pm 0.16^{\mathrm{a}}$ & $0.42 \pm 0.22^{\mathrm{a}}$ \\
\hline & & Dry Season & $0.57 \pm 0.19^{b}$ & $0.43 \pm 0.05^{\mathrm{b}}$ & $3.89 \pm 0.20^{\mathrm{a}}$ & $0.87 \pm 0.43^{b}$ \\
\hline & Decapoda & Dry Season & $0.35 \pm 0.08^{b}$ & $0.88 \pm 0.16^{\mathrm{b}}$ & $2.88 \pm 0.10^{\mathrm{a}}$ & $0.75 \pm 0.21^{\mathrm{b}}$ \\
\hline & \multirow{2}{*}{ Rotifera } & Wet Season & $0.75 \pm 0.12^{\mathrm{a}}$ & $0.64 \pm 0.13^{\mathrm{a}}$ & $1.58 \pm 0.28^{\mathrm{a}}$ & $0.48 \pm 0.11^{\mathrm{a}}$ \\
\hline & & Dry Season & $0.39 \pm 0.11^{\mathrm{b}}$ & $0.30 \pm 0.22^{\mathrm{b}}$ & $1.68 \pm 0.18^{\mathrm{a}}$ & $0.88 \pm 0.27^{b}$ \\
\hline \multirow{8}{*}{ Ejagham } & \multirow{2}{*}{ Cladocera } & Wet Season & $0.86 \pm 0.22^{\mathrm{a}}$ & $0.24 \pm 0.12^{\mathrm{a}}$ & $5.34 \pm 0.21^{\mathrm{a}}$ & $0.87 \pm 0.13^{\mathrm{a}}$ \\
\hline & & Dry Season & $0.47 \pm 0.13^{\mathrm{b}}$ & $0.55 \pm 0.11^{\mathrm{b}}$ & $4.99 \pm 0.31^{\mathrm{a}}$ & $0.48 \pm 0.11^{\mathrm{b}}$ \\
\hline & \multirow{2}{*}{ Copepoda } & Wet Season & $0.86 \pm 0.10^{\mathrm{a}}$ & $0.242 \pm 0.15^{\mathrm{a}}$ & $4.34 \pm 0.24^{\mathrm{a}}$ & $0.59 \pm 0.22^{\mathrm{a}}$ \\
\hline & & Dry Season & $0.45 \pm 0.25^{\mathrm{b}}$ & $0.64 \pm 0.14^{\mathrm{b}}$ & $4.33 \pm 0.3^{\mathrm{a}}$ & $0.97 \pm 0.33^{b}$ \\
\hline & \multirow{2}{*}{ Decapoda } & Wet Season & $0.75 \pm 0.15^{\mathrm{a}}$ & $0.22 \pm 0.19^{\mathrm{a}}$ & $3.12 \pm 0.22^{\mathrm{a}}$ & $0.46 \pm 0.13^{\mathrm{a}}$ \\
\hline & & Dry Season & $0.32 \pm 0.12^{\mathrm{b}}$ & $0.52 \pm 0.08^{\mathrm{b}}$ & $3.55 \pm 0.12^{\mathrm{a}}$ & $0.86 \pm 0.32^{b}$ \\
\hline & \multirow{2}{*}{ Rotifera } & Wet Season & $0.82 \pm 0.18^{\mathrm{a}}$ & $0.20 \pm 0.18^{\mathrm{a}}$ & $1.88 \pm 0.32^{\mathrm{a}}$ & $0.52 \pm 0.21^{\mathrm{a}}$ \\
\hline & & Dry Season & $0.54 \pm 0.21^{\mathrm{b}}$ & $0.50 \pm 0.16^{\mathrm{b}}$ & $2.44 \pm 0.21^{\mathrm{a}}$ & $0.97 \pm 0.17^{\mathrm{b}}$ \\
\hline
\end{tabular}

Table 6. Trophic state index due to chlorophyl-a, secchi disk and total phosphorus.

\begin{tabular}{|c|c|c|c|c|c|c|c|}
\hline \multirow[b]{2}{*}{ Lakes } & \multirow[b]{2}{*}{ Parameter } & \multicolumn{2}{|c|}{ Mean Values } & \multicolumn{2}{|c|}{ Trophic Points } & \multicolumn{2}{|c|}{ Classification } \\
\hline & & W & $\mathrm{D}$ & W & $\mathrm{D}$ & W & $\mathrm{D}$ \\
\hline \multirow{4}{*}{ Ikot Okpora } & Chlorophyll-a ( $\mu \mathrm{g} / \mathrm{L})$ & 1.77 & 2.54 & 31.11 & 41.21 & Oligotrophy & Mesotrophy \\
\hline & Secchi Disk (m) & 2.16 & 1.17 & 34.23 & 45.52 & Mesotrophy & Mesotrophy \\
\hline & Total Phosphorus $(\mu \mathrm{g} / \mathrm{L})$ & 0.05 & 0.18 & 51.25 & 60.9 & Eutrophy & Eutrophy \\
\hline & Chlorophyll-a ( $\mu \mathrm{g} / \mathrm{L})$ & 2.24 & 2.89 & 42.31 & 52.43 & Mesotrophy & Eutrophy \\
\hline \multirow[t]{3}{*}{ Obubra } & Secchi Disk (m) & 2.85 & 1.78 & 42.27 & 51.11 & Mesotrophy & Eutrophy \\
\hline & Total Phosphorus $(\mu \mathrm{g} / \mathrm{L})$ & 0.05 & 0.06 & 57.34 & 63.12 & Eutrophy & Eutrophy \\
\hline & Chlorophyll-a $(\mu \mathrm{g} / \mathrm{L})$ & 3.42 & 4.08 & 50.78 & 57.02 & Eutrophy & Eutrophy \\
\hline \multirow[t]{2}{*}{ Ejagham } & Secchi Disk (m) & 1.34 & 0.95 & 54.3 & 77.22 & Hypereutrophy & Eutrophy \\
\hline & Total Phosphorus $(\mu \mathrm{g} / \mathrm{L})$ & 0.09 & 0.11 & 70.21 & 74.4 & Hypereutrophy & Hypereutrophy \\
\hline
\end{tabular}




\subsection{Diversity}

Species diversity measured by Shannon Weaner index is directly proportional to the number of species in the sample and the uniformity of the species distribution in the total abundance [45]. In the three lakes species diversity was relatively high, which indicates good environmental conditions conducive to the development of many species, and according to Kajak [46] - moderate trophy of waters. It was only in wet season samples that values were much lower which was reflected by the lowest recorded number of species in the zooplankton community [47] as well as by the domination of single species, accompanied by low proportions of other taxa. This indicated poor lake conditions. This hypothesis that decrease in lake condition followed by community structure simplification was also confirmed by Rogozin [48].

\subsection{Trophic State Index (Tsi)}

Chlorophyll-a is used by all phytoplankton to capture sunlight for photosynthesis. Chlorophyll (Chl-a) concentration is a uniquely algal trait of the water column and function as a reliable measure of phytoplankton biomass [49]. Secchi depth is generally a good indicator of chlorophyll-a concentration and reported to be negatively related to chlorophyll-a concentration [50]. In Ejagham Lake, when minimum Secchi depth was observed, the highest chlorophyll-a value was recorded. However secchi disk visibility may not always be acceptable as an index of high productivity as some regions are affected by non-algal turbidity. In Ejagham and Obubra lakes, TSI (Chl-a) was found to be 70.02 and 52.43 respectively while TSI (SD) was 57.22 and 51.11 respectively and TSI value greater than 50 is usually associated with eutophy or high productivity [5]. For lakes that have a few rooted aquatic plants and little non-algal turbidity, the TSI (SD) and TSI (Chl-a) are approximately the same [6] as was observed in the Ikot Okpora and Obubra Lakes. Therefore, TSI (SD) is only useful to those lakes where turbidity is as a result of algal biomass [51]. In this study non algal turbidity was observed in Ejagham lake where the TSI (SD) was highest. Many factors are known to influence Secchi depth. The most important factors include primary production, the amount of resuspended material and the amount of coloured matter in the lake. Secchi depth do not depend solely on autochthonous lake production but also very much on allochthonous influences and resuspension [5]. So it is not correct to classify Ejagham lake as hypereutrophic on basis of secchi depth measurements. Same difference between TSI (SD) and TSI (Chl) values has been found for Sri Lankan reservoirs [52] and TSI (Chl) has been found to be a reliable means for quantifying trophic state at least for fisheries management purposes. Lake Ejagham can be characterized as eutrophic during both seasons on the basis of Chlorophyll-a concentration while Obubra Lake is eutrophic and mesotrophic during dry and wet seasons respectively. The Ikot Okpora Lake, however, show mesotrophic and oligotrophic characteristics in dry and wet seasons respectively. Oligotrophic lake generally host very little or no vegetation and is relatively clear while eutrophic lakes tend to host large quantities of organisms, including algal bloom. Each trophic class supports different types of fish and other organisms. If the algal biomass in a lake reaches very high level $(>80)$ fish kills occur as decomposing biomass deoxygenate the water. A lake situated in nutrient rich area may be naturally eutrophic. Nutrients carried into water bodies from non-point sources such as agricultural run-offs, residential fertilizers and sewage, will all increase biomass and can cause oligotrophic lake to become hypereutrophic.

The three lakes are at different stages of development and are influenced by season and location. Lake located at the savannah area of the floodplain eutrophies faster than lake at the forest section due to higher intensity of direct sunlight penetration and temperatures which increases photosynthesis and biodegradation respectively, in the former. The forest helps limit eutrophication due to the absence of anthropogenic inputs. The dry season trophic state of the tropical lakes seems to be more advanced because of the dilution factor by rain water. Lake Ikot Okpora may be recommended for an unfiltered water supply particularly during the wet season and is suitable for fisheries and recreation in all seasons. Obubra Lake may be suitable for fisheries but dominated mostly by tolerant species, Clariidae and Cichlidae families during the wet season while Ejagham Lake maybe have very few tolerant fish species and not suitable for recreation.

\section{REFERENCES}

[1] S. Kroeger, E. Fensin, K. Lynch and M. V. Borgh, "United State Water Programs that Use Algae as Biological Assessment Tool," Technical Guidance Document: EPA 007-841, 1999.

[2] D. F. Westlake, "The Functioning of the Freshwater Ecosystem," International Biological Programme, Cambridge University Press, London, Vol. 122, 2003, pp. 423-442.

[3] A. Payne, "Ecology of Tropical Lakes and Rivers," John Wiley and Sons, New York, 1986.

[4] G. M. Sechi and A. Sulis, "Multi-Reservoir System Optimization Using Chlorophyll-A Trophic Indexes," Water Resources Managemnet, Vol. 21, No. 5, 2007, pp. 849-860. doi:10.1007/s11269-006-9114-3

[5] L. Håkanson and V. V. Boulion, "Regularities in Primary 
Production, Secchi Depth and Fish Yield and a New System to Define Trophic and Humic State Indices for Lake Ecosystems," International Review Hydrobiology, Vol. 86,2001, pp. 23-62.

doi:10.1002/1522-2632(200101)86:1<23::AID-IROH23> 3.0.CO;2-4

[6] R. E. Carlson and J. Simpson, "A Coordinator's Guide to Volunteer Lake Monitoring Methods," North American Lake Management Society, Madison, 1996.

[7] L. Sifa and X. Senlin, "Culture and Capture of Fish in Chinese Reservoirs," IDRC, Ottawa, 1995.

[8] D. G. Frey, "Wisconsin: Birge-Juday Era," In: D. G. Frey Ed., Limnology in North America, The University of Wisconsin Press, Madison, 1963.

[9] S. D. Peckham, J. W. Chipman, T. M. Lillesand and S. I. Dodson, "Alternate Stable States and the Shape of the Lake Trophic Distribution," Hydrobiologia, Vol. 571, No. 1, 2006, pp. 401-407. doi:10.1007/s10750-006-0221-1

[10] I. C. Duggan, J. D. Green and R. J. Shiel, "Distribution of Rotifers in North Island, New Zealand, and Their Potential Use as Bioindicators of Lake Trophic State," Hydrobiologia, Vol. 446-447, No. 1, 2003, pp. 155-157.

[11] S. Guess, D. Albrecht, H. J. Krambeck, D. C. Mueller-Navarra and H. Mumm, "Impact of Weather on Lake Ecosystem, Assessed by Cyclo-Stationary MCCA of Long-Term Observations," Ecology, Vol. 81, No. 6, 2003, pp. 1720-1730.

[12] K. O. Coyle and A. I. Pinchuk, "Climate-Related Differences in Zooplankton Density and Growth on the Inner Shelf of the South-Eastern Bering Sea," Progress in Oceanography, Vol. 55, No. 1-2, 2002, pp. 177-179. doi:10.1016/S0079-6611(02)00077-0

[13] A. Hobaek, M. Manca and T. Anderson, "Factors Influencing Species Richness Lacustrine Zooplankton," Acta Oecologica, Vol. 23, No. 3, 2002, pp. 155-165. doi:10.1016/S1146-609X(02)01147-5

[14] G. Balvay, "Evolution du Zooplacton du Leman," Campagne 1999, Rapp. Comm. int. prot. Eaux. Leman conte pollut (CIPEL), 2000.

[15] M. G. Nogueira, "Phytoplankton Composition, Dominance and Abundance as Indicators of Environmental Compartmentalization in Jurumirim Reservoir (Paranapanema River)," Hydrobiologia, São Paulo, Vol. 431, No. 2-3, 2000, pp. 115-128. doi:10.1023/A:1003769408757

[16] C. C. Figueredo and A. Giani, "Seasonal Variation in the Diversity and Species Richness of Phytoplankton in a Tropical Eutrophic Reservoir," Hydrobiologia, Vol. 445, No. 3, 2001, pp. 165-174. doi:10.1023/A:1017513731393

[17] C. S. Pedrozo and O. Rocha, "Zooplankton and Water Quality of Lakes of the Northern Coast of Rio Grande Do Sul State, Brazil," Acta Limnologica Brasiliensia, Vol. 17, No. 4, 2005, pp. 445-459.

[18] E. I. L. Silva, "Ecology of Phytoplankton in Tropical Waters: Introduction to the Topic and Ecosystem Changes from Sri Lanka," Asian Journal of Water Environmental Pollution, Vol. 4, No. 1, 2005, pp. 25-35.
[19] D. Zorka, V. Mitrovic-Tutundzic, Z. Markovic and I. Zivic, "Monitoring Water Quality Using Zooplankton Organisms as Bioindicators at the Dubica Fish Farm, Serbia," Archives of Biological Sciences, Vol. 58, No. 4, 2006, pp. 245-248. doi:10.2298/ABS0604245D

[20] B. O. Offem and E. O. Ayotunde, "Toxicity of Lead to Freshwater Invertebrates. Waterfleas; Daphnia magna and Cyclop sp) in Fish Ponds in a Tropical Floodplain," Water Air Soil Pollution, Vol. 192, No. 1-4, 2008, pp. 39-46. doi:10.1007/s11270-008-9632-0

[21] B. O. Offem, Y. Akegbejo-Samsons, I. T. Omoniyi and G. U. Ikpi, "Dynamics of the Limnological Features and Diversity of Zooplankton Populations of the Cross River System SE Nigeria," Knowledge and Management of Aquatic Ecosystems, Vol. 393, No. 2, 2009, pp. 2-19. doi: $10.1051 / \mathrm{kmae} / 2009013$

[22] U. I. Enin, "The Artisanal Shrimp Fishery of the Outer Cross River Estuary, Nigeria," Ph. D. Thesis, University of Calabar, Calabar, 1994.

[23] H. I. Golterman, "Methods for Chemical Analysis of Freshwater," International Biological Programme, Handbook \& Oxford, Blackwell Scientific Publications, Oxford, 1969.

[24] American Public Health Association (APHA), "Standard Methods for the Examination of Water and Waste Water," 20th Edition, Washington DC, 1998.

[25] G. W. Prescott, "Algae of the Western Great Lakes Area," Brown Publishers, Dubuque, 1951.

[26] W. T. Edmondson, "Fresh Water Biology," 2nd Edition, John Wiley \& Sons Inc., New York, 1959.

[27] D. M. John, B. A. Whitton and A. J. Brook, "The Freshwater Algal Flora of the British Isles: An Identification Guide to Freshwater and Terrestrial Algae," 1st Edition, Cambridge University Press, Cambridge, 2002.

[28] R. A. Vollenweider, "A Manual on Methods for Measuring Primary Production in Aquatic Environments," 2nd Edition, Blackwell Scientific Publications, Oxford, 1974.

[29] C. E. Boyd, "Water Quality in Warm Water Fish Ponds," Auburn University Publishers, Auburn, 1981.

[30] M. O. Kadiri, "Seasonal Changes in the Phytoplankton Biomass of Shallow Tropical Reservoir, Nigeria," Journal of Botany, Vol. 6, 1988, pp. 167-173.

[31] E. C. Kemdirim, "Checklist of Phytoplankton of Shedam Reservoir in Plateau State, Nigeria," Journal of Aquatic Sciences, Vol. 16, 2001, pp. 61-66.

[32] E. R. Akpan and J. O. Offem, "Seasonal Variation in the Water Quality of Cross River, Nigeria," Hydrobiologia, Vol. 26, No. 2, 1993, pp. 95-98.

[33] A. E. Magurran, "Ecological Diversity and Its Measurement," Cambridge University Press, Cambridge, 1988.

[34] J. H. Zar, "Biostatistical Analysis," Pretice-Hall International, London, 1984.

[35] J. A. Downing and E. Mccauley, "The Nitrogen: Phosphorus Relationship in Lakes," Limnology Oceangraphy, Vol. 37, 1992. pp. 936-977.

doi:10.4319/10.1992.37.5.0936 
[36] B. Moss, J. Madgwick and G. Phillips, "A Guide to the Restoration of Nutrients-Enrich Shallow Lakes," W. W. Hawes, Suffolk, 1997.

[37] J. Padisak and C. S. Reynolds, "Selection of Phytoplankton Associations in Lake Balaton, Hungary in Response to Eutrophication and Restoration Measures, with Special Reference to the Cyanoprokaryotes," Hydrobiologia, Vol. 384, 1998, pp. 41-53. doi:10.1023/A:1003255529403

[38] M. P. Stoyneva, "Steady-State Phytoplankton Assemblage in Shallow Bulgarian Wetlands," Hydrobiologia, Vol. 502, No. 1-3, 2003, pp. 169-177. doi:10.1023/B:HYDR.0000004279.59719.7e

[39] C. Berger and H. E. Sweers, "Ijsselmeer and Its Phytoplankton with Special Attention to the Suitability of the Lake as a Habitat for Oscillatoria Agardhii Gom," Journal of Plankton Research, Vol. 10, No. 4, 1988, pp. 579-586. doi:10.1093/plankt/10.4.579

[40] D. I. Nwankwo, K. Onyema and T. A. Adesah, "A Survey of Harmful Algae in Coastal Waters of South Western Nigeria," Journal of Nigerian Environmental Society, Vol. 1, No. 2, 2003, pp. 241-246.

[41] J. Hall, J. B. Catharine and W. Carolyn, "Environmental Gradient and Zooplankton Distribution, in a Shallow, Tidal Lake," Archives of Hydrobiologia, Vol. 154, No. 3, 2002, pp. 485-490.

[42] A. Karabin, "Pelagic Zooplankton (Rotatoria and Crustacea) Variation in the Process of Lake Eutrophication. II. Modifying Effect of Biotic Agents," Polish Journal of Ecology, Vol. 33, No. 4, 1985b, pp. 567-589.

[43] S. Sendacz, E. Kubo and M. A. Cestarolli, "Limnologia de Reservatios do Estado do Sao Paulo, Brasil, VIII Zooplankton," Boletim do Instituto de Saúde, Vol. 12, 1985, pp. $145-176$

[44] A. Swierzoski, M. Godlewska and T. Poltorak, "The Relationship between the Spatial Distribution of Fish, Zooplankton and Other Environmental Parameters in the Solina Reservoir, Poland," Aquatic Living Resources, Vol.
13, 2000, pp. 373-387. doi:10.1016/S0990-7440(00)01085-8

[45] C. J. Krebs, "Ecology: The Experimental Analysis of Distribution and Abundance," Harper Intel, Lectual Edition, New York, 1996.

[46] Z. Kajak, "Ecological Characteristics of Lakes in North-Eastern Poland versus Their Trophic Gradient," Polish Journal of Ecology, Vol. 31, 1983, pp. 495-510.

[47] K. Irvine, M. T. Bales, B. Moss, J. H. Stanfield and D. Snook, "Trophic Relations in Hickling Broad-A Shallow and Brackish Eutrophic Lake," Verhandlungen des Internationalen Verein Limnologie, Vol. 24, 1990, pp. 576-587.

[48] A. G. Rogozin, "Specific Structural Features of Zooplankton in Lakes Differing in Trophic Status: Species Population," Ekologia, Moscow, Vol. 6, 2000, pp. 438-455.

[49] M. J. Behrenfeld and E. Boss, "Beam Attenuation and Chlorophyll Concentration as Alternative Optical Indices of Phytoplankton Biomass," Journal of Marine Research, Vol. 64, 2006, pp. 431-451. doi: $10.1357 / 002224006778189563$

[50] G. Almazan and C. E. Boyd, “An Evaluation of Secchi Disk Visibility for Estimating Plankton Density in Fish Ponds," Hydrobiologia, Vol. 61, No. 3, 1978, pp. 601608. doi:10.1007/BF00044446

[51] U. A. D. Jayasinghe, U. S. Amarasinghe and S. S. De Silva, "Trophic Classification of Non-Perennial Reservoirs Utilized for the Development of Culture-Based Fisheries, Sri Lanka," International Review of Hydrobiology, Vol. 90, No. 2, 2005, pp. 209-222. doi:10.1002/iroh.200410753

[52] F. Dominique, G. Hans, L. Malgorzata, C. Lawrence and K. Andoline, "Integrated Water Resource Management for Important Deep European Lakes and Their Catchment Areas," Eurolakes, Vol. 36, 2003, pp. 56-67. 